\title{
Deep Learning for Polarimetric Radar Quantitative Precipitation Estimation during Landfalling Typhoons in South China
}

\author{
Yonghua Zhang ${ }^{1,2,3}$, Shuoben Bi ${ }^{1, * \mathbb{D}}$, Liping Liu ${ }^{4}$, Haonan Chen ${ }^{5} \mathbb{D}$, Yi Zhang ${ }^{2}$, Ping Shen ${ }^{6}$, Fan Yang ${ }^{7}$, \\ Yaqiang Wang ${ }^{3}$, , Yang Zhang ${ }^{4}$ and Shun Yao ${ }^{5}$ \\ 1 School of Geographical Sciences, Nanjing University of Information Science \& Technology, \\ Nanjing 210044, China; zhangyh@gd121.cn \\ 2 Guangdong Meteorological Public Service Center, Guangzhou 510641, China; zhangy@gd121.cn \\ 3 Institute of Artificial Intelligence for Meteorology, Chinese Academy of Meteorological Sciences, \\ Beijing 100081, China; yqwang@cma.gov.cn \\ 4 State Key Laboratory of Severe Weather, Chinese Academy of Meteorological Sciences, Beijing 100081, China; \\ liulp@cma.gov.cn (L.L.); zhangyang_cams@cma.gov.cn (Y.Z.) \\ 5 Department of Electrical and Computer Engineering, Colorado State University, Fort Collins, CO 80523, USA; \\ haonan.chen@colostate.edu (H.C.); s.yao@colostate.edu (S.Y.) \\ 6 Guangdong Emergency Early Warning Release Center, Guangzhou 510641, China; shenping@gd121.cn \\ 7 Guangdong Technology Support Center of Flood Control, Guangzhou 510635, China; slt_yangfan@gd.gov.cn \\ * Correspondence: bishuoben@163.com; Tel.: +86-025-5873-1295
}

check for

updates

Citation: Zhang, Y.; Bi, S.; Liu, L.; Chen, H.; Zhang, Y.; Shen, P.; Yang, F.; Wang, Y.; Zhang, Y.; Yao, S. Deep Learning for Polarimetric Radar Quantitative Precipitation Estimation during Landfalling Typhoons in South China. Remote Sens. 2021, 13, 3157. https://doi.org/10.3390/ rs13163157

Academic Editor: Kenji Nakamura

Received: 20 July 2021

Accepted: 7 August 2021

Published: 10 August 2021

Publisher's Note: MDPI stays neutral with regard to jurisdictional claims in published maps and institutional affiliations.

Copyright: (c) 2021 by the authors. Licensee MDPI, Basel, Switzerland. This article is an open access article distributed under the terms and conditions of the Creative Commons Attribution (CC BY) license (https:/ / creativecommons.org/licenses/by/ $4.0 /)$.
Abstract: Heavy rain associated with landfalling typhoons often leads to disasters in South China, which can be reduced by improving the accuracy of radar quantitative precipitation estimation (QPE). At present, raindrop size distribution (DSD)-based nonlinear fitting (QPE ${ }_{D S D}$ ) and traditional neural networks are the main radar QPE algorithms. The former is not sufficient to represent the spatiotemporal variability of DSDs through the generalized Z-R or polarimetric radar rainfall relations that are established using statistical methods since such parametric methods do not consider the spatial distribution of radar observables, and the latter is limited by the number of network layers and availability of data for training the model. In this paper, we propose an alternative approach to dual-polarization radar QPE based on deep learning (QPENet). Three datasets of “dual-polarization radar observations—surface rainfall (DPO—SR)" were constructed using radar observations and corresponding measurements from automatic weather stations (AWS) and used for QPENet $_{\mathrm{V} 1}, \mathrm{QPENet}_{\mathrm{V} 2}$, and $\mathrm{QPENet}_{\mathrm{V} 3}$. In particular, $13 \times 13,25 \times 25$, and $41 \times 41$ radar range bins surrounding each AWS location were used in constructing the datasets for QPENet 1 , QPENet ${ }_{V 2}$, and QPENet ${ }_{V 3}$, respectively. For training the QPENet models, the radar data and AWS measurements from eleven landfalling typhoons in South China during 2017-2019 were used. For demonstration, an independent typhoon event was randomly selected (i.e., Merbok) to implement the three trained models to produce rainfall estimates. The evaluation results and comparison with traditional QPE $\mathrm{DSD}$ algorithms show that the QPENet model has a better performance than the traditional parametric relations. Only when the hourly rainfall intensity is less than $5 \mathrm{~mm}\left(R<5 \mathrm{~mm} \cdot \mathrm{h}^{-1}\right)$, the QPE $\mathrm{DSD}$ model shows a comparable performance to QPENet. Comparing the three versions of the QPENet model, QPENet $t_{\mathrm{V} 2}$ has the best overall performance. Only when the hourly rainfall intensity is less than $5 \mathrm{~mm}\left(R<5 \mathrm{~mm} \cdot \mathrm{h}^{-1}\right)$, QPENet $\mathrm{V} 3$ performs the best.

Keywords: polarimetric radar; quantitative precipitation estimation; deep learning; convolutional neural network; landfalling typhoons

\section{Introduction}

Heavy rain from landfalling typhoons is one of the major natural disasters in South China, which often causes life and economic losses [1]. High-resolution precipitation estimation is a prerequisite for the typhoon rainfall forecast. The polarimetric radar (PR) is 
an efficient tool for observing precipitation and its microphysical structures [2]. The PR has significant advantages in quantitative precipitation estimation (QPE) over the singlepolarization radar due to enhanced observations of the precipitation particle size, shape, and orientation [3].

QPE algorithms have been studied for many years using the PR measurements. In principle, the quantitative relation between the surface rainfall and radar observation aloft can be obtained from measurements [3]. However, it is difficult to present this functional relation in a simple form due to the complex spatiotemporal variability in DSDs $[4,5]$, especially during typhoon events which are often characterized by complicated precipitation microphysical processes [6]. At present, DSD-based nonlinear fitting algorithms (hereafter referred to as the QPE approach [8-11] are the main QPE algorithms. The precipitation estimation accuracy of the parametric QPE $E_{D S D}$ methods is determined by the physical model of DSD and the relationship between the physical model and the radar parameters [2,12,13]. DSD characteristics vary with the location and precipitation type. The parameter relationships between rain rate $(R)$ and the polarimetric radar observables, including reflectivity $\left(Z_{\mathrm{H}}\right)$, differential reflectivity $\left(Z_{\mathrm{DR}}\right)$, and the specific differential phase $\left(K_{\mathrm{DP}}\right)$, are not sufficient to characterize the variation [14-17]. In addition, the traditional neural network method is limited by the number of network layers and the availability of training data, resulting in poor learning performance.

In recent years, deep learning has been widely used in the fields of computer vision $[18,19]$ and geoscience including precipitation research (e.g., $[20,21])$. Tan et al. built a deep neural network to estimate precipitation based on radar and surface rainfall observation data [22,23]. Chen et al. proposed a neural network based on a fusion mechanism to improve satellite precipitation retrievals using ground radar estimates [24]. Chandrasekar et al. [25] and Chen et al. [3] constructed a precipitation estimation algorithm composed of two deep neural networks in order to establish the relationship between a spaceborne radar and rain gauges using a ground radar as a bridge. Moraux et al. proposed a deep-learning precipitation estimation method based on satellite and rain gauge observations [26]. The abovementioned studies show that deep-learning algorithms have great potential for improving radar-based QPE.

In this study, we extended the deep learning applications for radar QPE in south China, with an emphasis on typhoon events. This study used hybrid volume scan data of $Z_{\mathrm{H}}$, $Z_{\mathrm{DR}}$, and $K_{\mathrm{DP}}$ for precipitation estimation, which can better represent the actual cloud and rainfall conditions and help reduce systematic biases. In particular, a deep convolutional neural network (CNN) composed of multiscale convolutional operations was designed to realize the complex nonlinear mapping from radar measurements to rainfall rate. This multiscale encoding-decoding network structure had strong feature extraction and fitting capability, as well as the invariance of displacement, scale, and deformation. It could better extract the spatial characteristics of precipitation from radar data and learn the relationship between multidimensional radar observations and surface rainfall. In addition, for the same $\mathrm{CNN}$ structure, we used three different datasets to quantify the areal representation of radar observables and address which range bins should be used to obtain reliable precipitation estimates. In particular, $13 \times 13,25 \times 25$, and $41 \times 41$ radar range bins surrounding each AWS location were used in constructing the three datasets. For training the QPENet models, the radar data and AWS measurements from eleven landfalling typhoons in South China during 2017-2019 were used. For demonstration, an independent typhoon event was randomly selected (i.e., Merbok) to evaluate the trained models and compare with the traditional QPE $\mathrm{DSD}_{\mathrm{D}}$ algorithms.

The remainder of this paper is organized as follows. Section 2 introduces the datasets used in this study. Section 3 details the deep learning-based radar QPE methodology, including the model architecture and training strategy, as well as how to construct the model input data for machine learning. In Section 4, the precipitation estimation accuracy of the three trained models is evaluated, and the influence of the input data dimension on the 
model results is thoroughly discussed. Section 5 compares the data with the conventional radar QPE algorithm while Section 6 summarizes the main findings of this research.

\section{Data}

Eleven heavy rainfall events caused by landfalling typhoons in South China from 2017 to 2019 were used in this study. Figure 1 shows the tracks of the eleven typhoons and the locations of the Guangzhou radar and nearby surface automatic weather stations (AWS). Table 1 lists the detailed information of these eleven typhoon events.

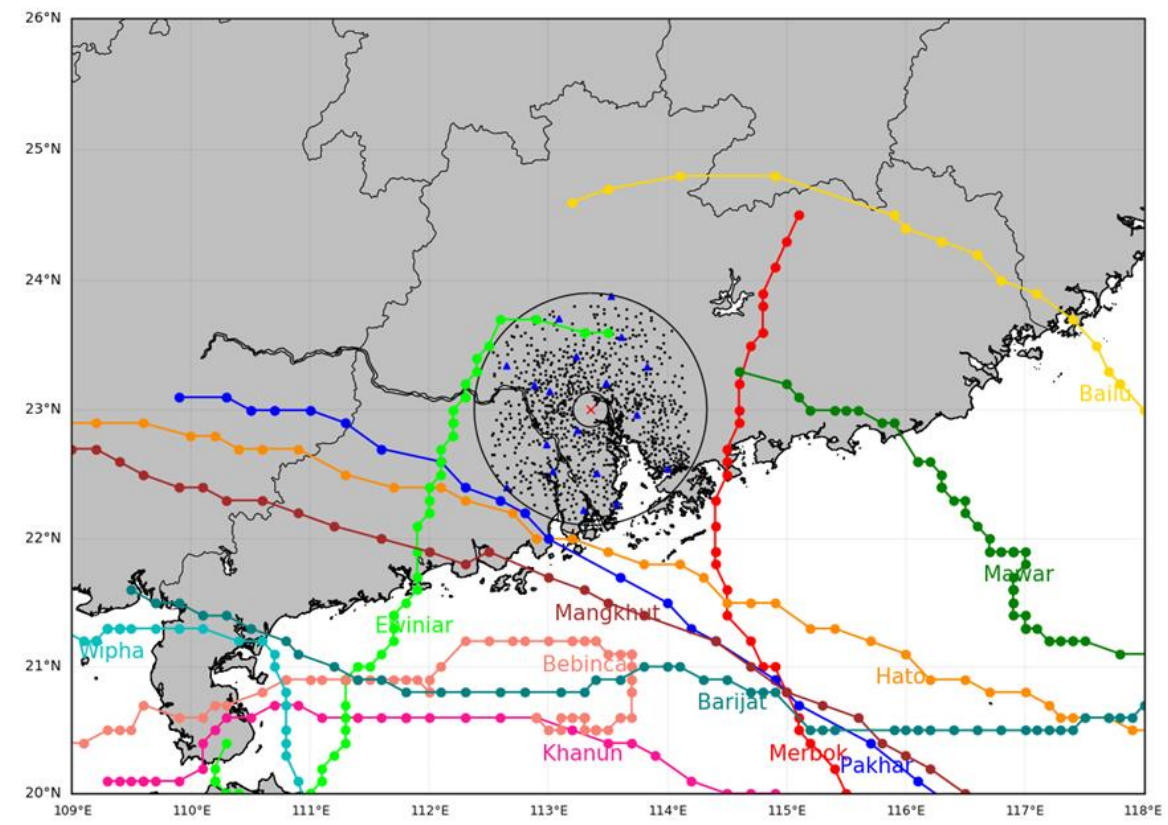

Figure 1. Tracks of the eleven landfalling typhoons and the locations of the Guangzhou radar (red cross) and automatic weather stations. The blue triangles and black dots denote the national automatic weather stations and regional automatic weather stations between the $15 \mathrm{~km}$ and $100 \mathrm{~km}$ coverage ranges (two black circles) of the Guangzhou radar.

Table 1. Detailed information on the eleven typhoon precipitation events.

\begin{tabular}{|c|c|c|c|c|c|c|c|}
\hline$\#$ & $\begin{array}{l}\text { Name } \\
\text { (No.) }\end{array}$ & Date (UTC) & $\begin{array}{l}\text { Total } \\
\text { Time } \\
\text { (h) }\end{array}$ & $\begin{array}{l}\text { No. of } \\
\text { Valued } \\
\text { gauges }\end{array}$ & $\begin{array}{c}\text { No. of } \\
\text { Radar } \\
\text { Volumes }\end{array}$ & $\begin{array}{c}\text { Mean Gauge } \\
\text { Accumulation } \\
(\mathrm{mm})\end{array}$ & $\begin{array}{l}\text { Maximum Gauge } \\
\text { Accumulation } \\
(\mathrm{mm})\end{array}$ \\
\hline 1 & $\begin{array}{c}\text { Merbok } \\
(1702)\end{array}$ & June 12-13, 2017 & 19 & 544 & 190 & 18.32 & 144.4 \\
\hline 2 & $\begin{array}{l}\text { Hato } \\
(1713)\end{array}$ & August 23, 2017 & 12 & 775 & 120 & 25.22 & 54 \\
\hline 3 & $\begin{array}{l}\text { Pakhar } \\
(1714)\end{array}$ & August 26-27, 2017 & 9 & 763 & 90 & 44.54 & 71 \\
\hline 4 & $\begin{array}{l}\text { Mawar } \\
(1716)\end{array}$ & September 02-04, 2017 & 28 & 720 & 280 & 31.67 & 211.3 \\
\hline 5 & $\begin{array}{l}\text { Khanun } \\
(1720)\end{array}$ & October 15-16, 2017 & 18 & 765 & 180 & 27.33 & 85.2 \\
\hline 6 & $\begin{array}{c}\text { Ewiniar } \\
(1804)\end{array}$ & June 07-08, 2018 & 37 & 793 & 370 & 212.46 & 311.2 \\
\hline 7 & $\begin{array}{c}\text { Bebinca } \\
(1816)\end{array}$ & August 10-15, 2018 & 119 & 804 & 1190 & 100.48 & 255.6 \\
\hline 8 & $\begin{array}{c}\text { Mangkhut } \\
(1822)\end{array}$ & September 16, 2018 & 9 & 797 & 90 & 77.44 & 148.4 \\
\hline 9 & $\begin{array}{l}\text { Barijat } \\
(1823)\end{array}$ & September 12-13, 2018 & 21 & 613 & 210 & 2.98 & 14 \\
\hline 10 & $\begin{array}{l}\text { Wipha } \\
\text { (1907) }\end{array}$ & August 01-02, 2019 & 20 & 806 & 200 & 44.62 & 170.4 \\
\hline 11 & $\begin{array}{l}\text { Bailu } \\
\text { (1911) }\end{array}$ & August 24-25, 2019 & 23 & 798 & 230 & 45.49 & 99.1 \\
\hline
\end{tabular}




\subsection{Dual-Polarization Radar Data}

In 2016, the Guangzhou radar was upgraded with dual-polarization capability. According to the standards of the China Meteorological Administration (CMA), this radar has been calibrated by the manufacturer [27]. After the dual-polarization upgrade, the Meteorological Observation Center of the CMA conducted a series of tests, such as the built-in system component test and routine calibrations. The test results show that the measurement uncertainty of $Z_{\mathrm{H}}$ is less than $1 \mathrm{~dB}$, and the measurement uncertainty of $Z_{\mathrm{DR}}$ is less than $0.4 \mathrm{~dB}$. The radar operates with the general volume coverage pattern 21 , which scans every six minutes with nine elevation angles $\left(0.5^{\circ}, 1.5^{\circ}, 2.4^{\circ}, 3.3^{\circ}, 4.3^{\circ}, 6.0^{\circ}, 9.9^{\circ}\right.$, $14.6^{\circ}$, and $19.5^{\circ}$ ). The radar beamwidth is $0.95^{\circ}$, the radial resolution is $0.25 \mathrm{~km}$, and the commonly used maximum detection distance is $230 \mathrm{~km}$.

In this study, polarimetric radar data in the range of $15-100 \mathrm{~km}$ from the radar (see Figure 1) were used in order to reduce the influence of the zero-degree layer and the interference of near-range ground objects. The selected eleven typhoon events consisted of 3150 volume scans, and the numbers of scans for each typhoon event are detailed in Table 1. In addition, thorough quality control was performed on the collected radar data, including removal of outliers and non-meteorological echoes, differential phase $\Phi_{\mathrm{DP}}$ processing, $K_{\mathrm{DP}}$ estimation, etc. [28]. The quality-controlled data were used as the input to the deep-learning model for precipitation estimation.

\subsection{Automatic Weather Station (AWS) Data}

In this study, the rainfall observations from 18 national and 1041 regional automatic weather stations were used as target labels in training the deep-learning model. The locations of the AWS relative to the Guangzhou radar are indicated in Figure 1. The AWS rainfall resolution is $0.1 \mathrm{~mm}$, and the rainfall data is archived every five minutes, including one-minute rainfall and five-minute accumulated rainfall. The six-minute accumulated rainfall can be obtained through the one-minute rainfall observation in order to match the radar observation times. Here, it should be noted that the method of Gou et al. [29] was used to control the quality of rainfall observations from the AWS and remove the suspicious records.

\section{Methods}

To test our deep-learning QPE method, the dataset was divided into two independent parts: the training dataset and the test dataset. The former was used to train the model by optimizing the learnable parameters of the model through the back-propagation algorithm [21]. In order to ensure that our model did not overfit the training data and could at the same time make full use of the existing data, we used the $k$-fold cross-validation method to optimize the network structure and training strategy through the performance evaluation of the $k$-fold validation dataset. The model and training strategy that performed the best on the validation dataset may overfit the dataset. Therefore, an independent test dataset was required for the final evaluation of the model.

In the following section, we describe in more detail how the data were prepared before being fed to our model, how the dataset was made for the training model, and how we split the data into the training dataset and the test dataset. Furthermore, we present the architecture of our model and its training strategy.

\subsection{Model Inputs}

The input features of the QPENet model are the dual-polarization radar-based data, and the target labels are rainfall measurements from the surface automatic weather stations. The QPENet model needs to be trained by datasets with tags. The model inputs used by Chen et al., the CAPPI reflectivity profiles with the horizontal resolution of $1 \mathrm{~km}$ and heights of 1, 2, 3, and $4 \mathrm{~km}$, were not used in our model [3]. To preserve the original radar information in the network and include the polarization quantities closely related to precipitation microphysics, this study proposed using multiple polarimetric observables 
from the hybrid scan strategy as the inputs. In order to construct the dataset, we should have first found the corresponding relationship between the dual-polarization radar data and surface rainfall. Properly connecting the surface rainfall values (target labels) with multidimensional radar measurements was critical to training the machine-learning model for enhanced precipitation estimates.

In particular, at a given time and AWS location, we built the matching samples between rainfall measurements from the AWS and polarimetric variables at the elevation angles and different ranges surrounding the AWS. A trustworthy data sample (Figure 2) was obtained through adjusting the radar observation range (range bins surrounding the AWS), observation height (elevation angles), and polarization quantity $\left(Z_{\mathrm{H}}, Z_{\mathrm{DR}}\right.$, and $\left.K_{\mathrm{DP}}\right)$.

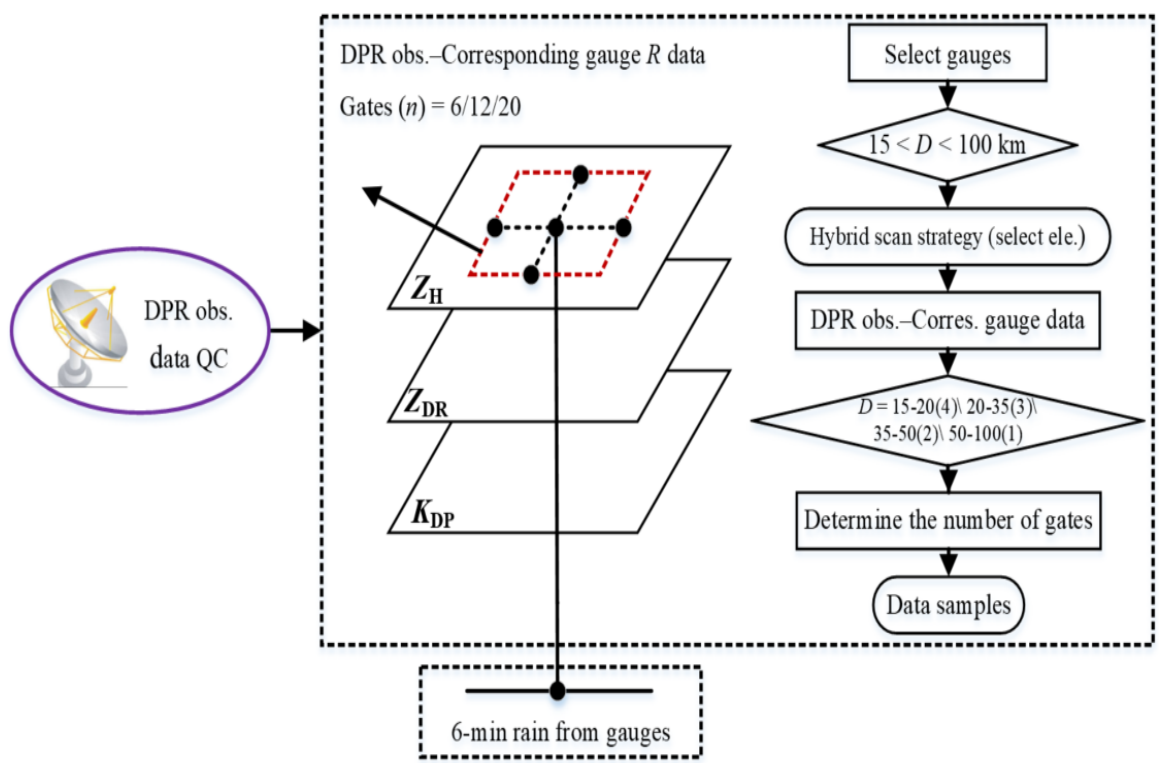

Figure 2. Construction of data samples for machine learning. $D$ is the distance from the automatic weather stations to the radar; (4), (3), (2), and (1) represent the fourth, third, second, and first scan elevation angles of the radar, respectively.

The specific steps for establishing the data sample and dataset of "DPO-SR" are as follows (Figure 2):

(1) Automatic weather station selection: To avoid the influence of the melting layer and ground clutter, only the automatic weather stations within $15-100 \mathrm{~km}$ of the radar with rainfall greater than $0.1 \mathrm{~mm}$ were used in this study.

(2) Hybrid scan: It is formed by the four lowest elevation angles. To avoid the effect of ground clutter, the fourth elevation angle $\left(3.3^{\circ}\right)$ was selected for $15-20 \mathrm{~km}$ away from the radar, the third elevation angle $\left(2.4^{\circ}\right)$ was selected for $20-35 \mathrm{~km}$, the second elevation angle $\left(1.5^{\circ}\right)$ was selected for $35-50 \mathrm{~km}$, and the first elevation angle $\left(0.5^{\circ}\right)$ was selected for $50-100 \mathrm{~km}$.

(3) Location correspondence: According to the distance from the automatic weather station to the radar, the range bin closest to the station at the corresponding elevation angle was selected as the corresponding range bin.

(4) Range bin selection: The range bin matrix of $(2 n+1) \times(2 n+1)$ was composed of the front and back $n$ range bins in the same radial direction of the corresponding range bin and the right and left $n$ radial directions of the corresponding range bins at the selected elevation angle.

(5) Generation of data samples: The matrix composed of the polarization quantities within the radar observation range bin (called data) and the observed rainfall amount (called label) from the corresponding automatic weather station was generated as a sample. 
(6) Steps (1)-(5) are the process of labeling (tagging) the rainfall from automatic weather stations for dual-polarization radar observations. The "dual-polarization radar observation-surface rainfall" dataset was generated by repeating the tagging process.

Generally, the wind is stronger in typhoon precipitation. Affected by the wind, the range of falling raindrops is relatively wide. In other words, a wider range of radar observations is related to the rainfall observed by surface automatic stations. In order to obtain a better correspondence between the dual-polarization radar observations and surface rainfall, a sample and the dataset of "DPO-SR" were established, which are more conducive to precipitation estimation; $13 \times 13,25 \times 25$, and $41 \times 41$ bins surrounding the range bin on top of the AWS were chosen as the radar observation range bins, that is, the radar observation areas roughly at $1.5 \mathrm{~km}, 3 \mathrm{~km}$, and $5 \mathrm{~km}$ away from the surface automatic weather stations were selected to correspond to the surface rainfall, and the QPE datasets (see Table 2 for details) for version $1(13 \times 13)$, version $2(25 \times 25)$, and version $3(41 \times 41)$ of the eleven typhoon events were respectively established to analyze the influence of the radar observation range on the typhoon precipitation estimation.

Table 2. Sample information of the "DPO—SR" dataset.

\begin{tabular}{ccccc}
\hline$\#$ & $\begin{array}{c}\text { Name } \\
\text { (No.) }\end{array}$ & $\begin{array}{c}\text { No. of Samples } \\
\text { in Dataset V1 }\end{array}$ & $\begin{array}{c}\text { No. of Samples } \\
\text { in Dataset V2 }\end{array}$ & $\begin{array}{c}\text { No. of Samples } \\
\text { in Dataset V3 }\end{array}$ \\
\hline 1 & Merbok (1702) & 4500 & 4502 & 4510 \\
2 & Hato (1713) & 19,196 & 19,188 & 19,198 \\
3 & Pakhar (1714) & 30,398 & 30,401 & 30,405 \\
4 & Mawar (1716) & 8995 & 8992 & 8993 \\
5 & Khanun (1720) & 11,892 & 11,880 & 11,884 \\
6 & Ewiniar (1804) & 115,703 & 115,737 & 115,726 \\
7 & Bebinca (1816) & 63,818 & 63,874 & 63,877 \\
8 & Mangkhut (1822) & 47,230 & 47,220 & 47,229 \\
9 & Barijat (1823) & 1412 & 1412 & 1414 \\
10 & Wipha (1907) & 32,097 & 32,099 & 32,089 \\
11 & Bailu (1911) & 39,932 & 39,935 & 39,942 \\
& Total & 375,173 & 375,240 & 375,267 \\
\hline
\end{tabular}

\subsection{Model Architecture}

The invariance of $\mathrm{CNN}$ displacement, scale, and deformation was used to extract the spatial features of radar data to better fit the relationship between radar observations and surface rainfall and give full play to the excellent feature extraction and fitting performance of the deep neural network (DNN). QPENet training and estimation processes (Figure 3) were designed to estimate the precipitation based on the classic DNN and CNN architectures, modules, and concepts (such as AlexNet [30], GoogLeNet [31], and ResNet [32]). The QPENet model is a regression deep CNN architecture directly facing the automatic weather station rainfall. It takes radar observation data as the inputs, automatic weather station rainfall as the target data, and mean square error as the loss function. The features in the inception module were used to extract multiscale precipitation features through multiple convolution kernels with different sizes. In particular, radar observations and the corresponding surface automatic weather station precipitation were used to train the QPENet regression network (the lower panel in Figure 3). The trained model could estimate surface precipitation based on the new radar observations (the upper panel in Figure 3) and evaluate its estimation accuracy through rainfall observation at new automatic weather stations. 


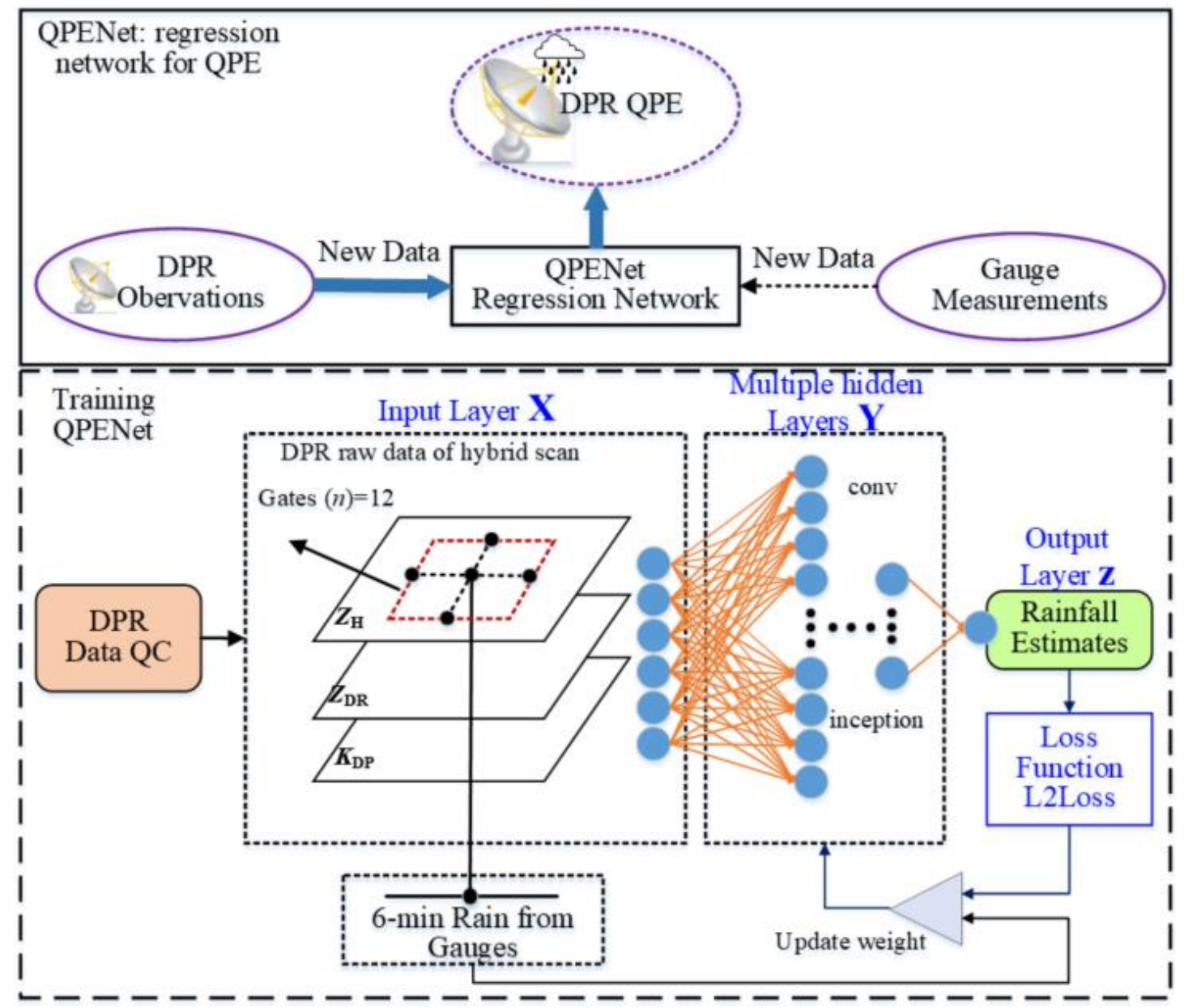

Figure 3. Simplified flowchart of the QPENet model structure and its application for polarimetric radar quantitative precipitation estimation.

The general expression of the QPENet model is as follows [3].

$$
\begin{gathered}
\boldsymbol{y}_{1}=f\left(\mathbf{w}_{1} \mathbf{X}+\mathbf{b}_{1}\right) \\
\boldsymbol{y}_{n}=f\left(\mathbf{w}_{n} \boldsymbol{y}_{n-1}+\mathbf{b}_{n}\right) \\
\mathbf{Z}=f\left(\mathbf{w}_{n+1} \boldsymbol{y}_{n}+\mathbf{b}_{n+1}\right)
\end{gathered}
$$

where $\mathbf{X}$ is the input variable which refers to multiple polarization parameters (i.e., $Z_{\mathrm{H}}$, $Z_{\mathrm{DR}}$, and $K_{\mathrm{DP}}$ ) of the radar at different ranges (i.e., $1.5 \mathrm{~km}, 3 \mathrm{~km}$, and $5 \mathrm{~km}$.); $y_{1} \ldots y_{n}$ are the outputs of the hidden layers; $\mathbf{w}_{1}$ is the weight vector for the input data; $\mathbf{w}_{2} \ldots \mathbf{w}_{n+1}$ are the weights of the $n$ hidden layers; $\mathbf{b}_{1} \ldots \mathbf{b}_{n+1}$ are the biases associated with the input layers and the hidden layers; $\mathbf{Z}$ is the output (i.e., precipitation estimation) compared with the target labels (i.e., AWS-measured rainfall) to update the weights.

Our problem was very similar to the semantic segmentation application, and the purpose of the latter is to assign each pixel in the image to a category. For example, the hourglassshaped network (HSN) is a kind of a convolutional neural network which can analyze the multiscale input, showing a good performance in the semantic segmentation of aerial images [33]. Due to the abovementioned reasons, our model could be divided into two parts, the encoder and the decoder (Figure 4). The encoder gradually reduced the input resolution through its maximum pooling layer, allowing the convolution kernel of the next layer to cover a larger spatial range. In addition, we used inception layers to further improve the multiscale conversion capability of the model [31] (Figure 5 and Table 3). These layers were composed of convolutional layers with different convolution kernel sizes to perform conversions of 
different scales. The decoder followed a pattern similar to the encoder. After the third maximum pooling layer, the transposed convolution was used instead of the maximum pooling layer to increase the resolution gradually. In this way, our model recovered the details lost in the continuous maximum pooling layer, reaching the highest resolution at the input time. Finally, the rain rate $\left(R\right.$; units: $\left.\mathrm{mm} \cdot \mathrm{h}^{-1}\right)$ was obtained through the GlobalAvgPool2D, Dropout, and Dense layers at the end of the decoder.

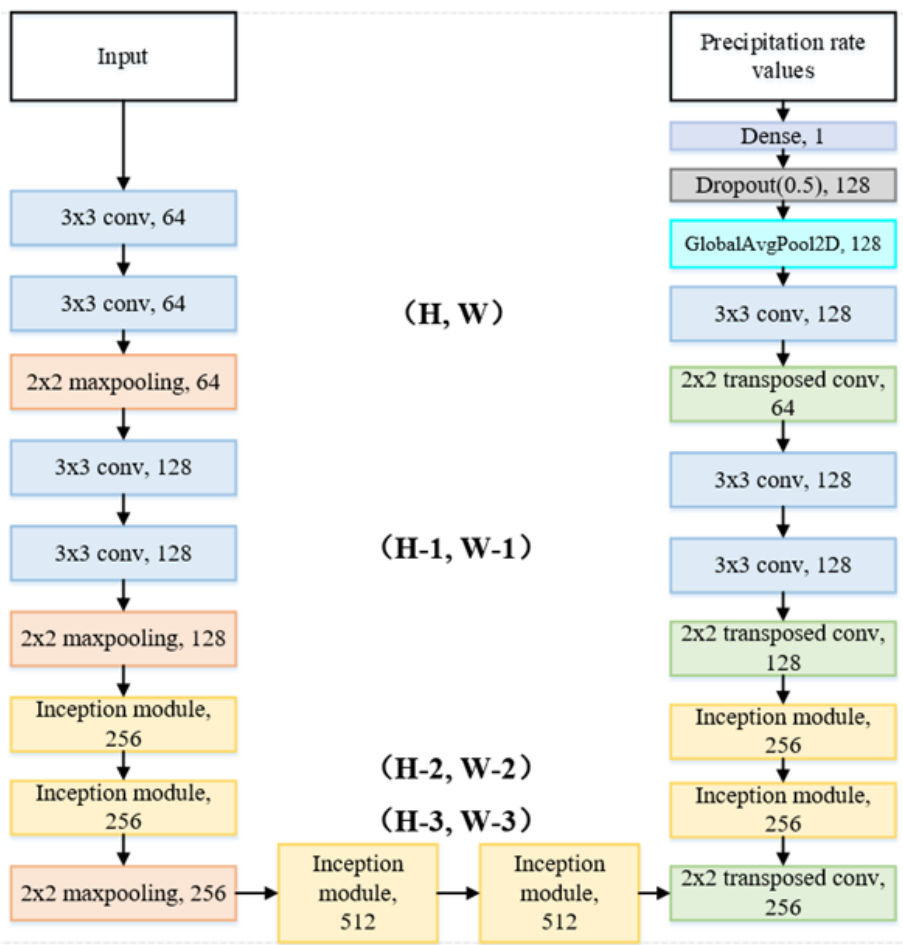

Figure 4. Detailed architecture of the proposed model. Each convolutional layer is followed by a batch normalization layer, and the ReLU is used as the activation function.

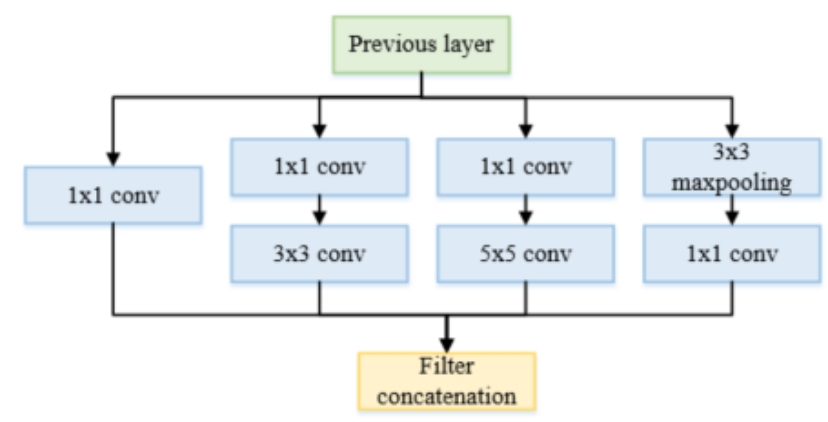

Figure 5. Diagram of the Inception module. The filter numbers used for each layer are given in Table 3.

Table 3. Filter numbers for each convolution type in the Inception module. Note: " $n \times n$ Reduce" stands for the $1 \times 1$ convolutions preceding the corresponding $n \times n$ convolutions.

\begin{tabular}{ccccccc}
\hline Total \# (Filter Output) & \# $\mathbf{1} \times \mathbf{1}$ & \# $\mathbf{3} \times \mathbf{3}$ Reduce & $\mathbf{\#} \mathbf{3} \mathbf{3}$ & \# $\mathbf{5} \times \mathbf{5}$ Reduce & \# $\mathbf{5} \times \mathbf{5}$ & \# $\mathbf{1} \times \mathbf{1}$ \\
\hline 256 & 64 & 128 & 128 & 64 & 32 & 32 \\
512 & 65 & 256 & 384 & 64 & 32 & 32 \\
\hline
\end{tabular}




\subsection{Training Strategies}

To train the model, we randomly chose one of the eleven typhoon precipitation events as the test dataset and the remaining ten as the training (validation) dataset. The $\mathrm{k}$-fold cross-validation method randomly selected nine of the ten typhoons as the training dataset and one as the validation dataset. After ten training sessions, the hyperparameter tuning of the network based on the network performance of the verification dataset was determined. To prevent the model from overfitting, the test dataset was used to evaluate its generalization ability.

To explore the influence of the radar observation range on the precipitation estimation, three versions $\left(V_{1}, V_{2}\right.$, and $\left.V_{3}\right)$ of the QPE dataset were used to train the model. The numerical stability of deep-learning model training is related to the quality of the training results [31]. In order to ensure the numerical stability of the training process and obtain better training results, we normalized the input data (radar observation) and target data (automatic weather station precipitation) so that their mean value was 0 , variance was 1 , and their values were between 0 and 1 .

Finally, based on the deep learning library of MXNet/Gluon from Amazon, the model selected Xavier for parameter initialization [34]. The Adam optimization algorithm with a learning rate of $10^{-3}$ and the method of early stopping were adopted, and four Nvidia Tesla P100s were used for training. The specific hyperparameter, the convergence epochs of our model, and the time spent on each batch are shown in Table 4.

Table 4. The hyperparameters of the QPENet model.

\begin{tabular}{cccccc}
\hline Dataset Version & Batch Size & Convergence Epochs & Learning Rate & Time per Epoch (s) & Weight Decay \\
\hline $\mathrm{V}_{1}$ & 512 & 2 & 0.001 & 114.5 & 0.0001 \\
$\mathrm{~V}_{2}$ & 512 & 12 & 0.001 & 231.2 & 0.0001 \\
$\mathrm{~V}_{3}$ & 256 & 26 & 0.001 & 615.4 & 0.0001 \\
\hline
\end{tabular}

\subsection{Evaluation Method}

Based on the test dataset, the automatic weather station rainfall was taken as the reference, and then the 6-min radar data was input into the network. By accumulating the output, the hourly rainfall intensity could be obtained. Furthermore, five indicators of correlation coefficient (CC), root-mean-square error (RMSE), normalized relative bias (NB), normalized absolute error (NE), and bias ratio were used to evaluate the estimation accuracy comprehensively. The results were then compared with the results of the QPEDSD method.

$$
\begin{aligned}
& C C=\frac{\sum_{i=1}^{n}\left(R A_{i}^{\text {radar }}-\overline{R_{i}^{\text {radar }}}\right)\left(R A_{i}^{\text {gauge }}-\overline{R_{i}^{\text {gauge }}}\right)}{\sqrt{\sum_{i=1}^{n}\left(R A_{i}^{\text {radar }}-\overline{R A_{i}^{\text {radar }}}\right)^{2} \sum_{i=1}^{n}\left(R A_{i}^{\text {gauge }}-\overline{R A_{i}^{\text {gauge }}}\right)^{2}}} \\
& \text { RMSE }=\sqrt{\frac{\sum_{i=1}^{\mathrm{n}}\left(\mathrm{RA}_{\mathrm{i}}^{\text {radar }}-\mathrm{RA}_{\mathrm{i}}^{\text {gauge }}\right)^{2}}{\mathrm{n}}} \\
& \mathrm{NB}=\frac{\sum_{\mathrm{i}=1}^{\mathrm{n}}\left(\mathrm{RA}_{\mathrm{i}}^{\text {radar }}-\mathrm{RA}_{\mathrm{i}}^{\text {gauge }}\right)}{\sum_{\mathrm{i}=1}^{\mathrm{n}} \mathrm{R}_{\mathrm{i}}^{\mathrm{DSD}}} \times 100 \\
& \mathrm{NE}=\frac{\sum_{\mathrm{i}=1}^{\mathrm{n}}\left|\mathrm{RA}_{\mathrm{i}}^{\text {radar }}-\mathrm{RA}_{\mathrm{i}}^{\text {gauge }}\right|}{\sum_{\mathrm{i}=1}^{\mathrm{n}} \mathrm{RA}_{\mathrm{i}}^{\text {gauge }}} \times 100 \\
& \text { bias ratio }=\frac{\sum_{i=1}^{n} R A_{i}^{\text {radar }}}{\sum_{i=1}^{n} R A_{i}^{\text {gauge }}}
\end{aligned}
$$


In the equations above, RA is the one-hour accumulated rainfall from the radar or automatic stations, and the overline indicates the average value; $\mathrm{n}$ is the number of $\mathrm{RA}_{\mathrm{i}}{ }^{\text {gauge }}-\mathrm{RA}_{\mathrm{i}}{ }^{\text {radar }}$ pairs; the units of measurement of the RMSE are millimeters; NE and $\mathrm{NB}$ are both percentages; and the bias ratio greater (or less) than 1 indicates overestimation (or underestimation).

\section{Results}

In this section, one typhoon from the eleven typhoon events in South China in 2017-2019 was randomly chosen to examine the QPENet algorithm for the operational Guangzhou radar. The Merbok (1702) typhoon event was taken as an example, and the $\mathrm{QPE}_{\mathrm{DSD}}$ and QPENet ${ }_{\mathrm{V} 2}$ algorithms were used to estimate the hourly average rainfall intensity (Figure 6) and accumulated rainfall intensity of this event. Figures 6 and 7 show that the spatial distributions of hourly average rainfall intensity and accumulated rainfall intensity of these two estimation algorithms were basically the same, both consistent with actual precipitation events.
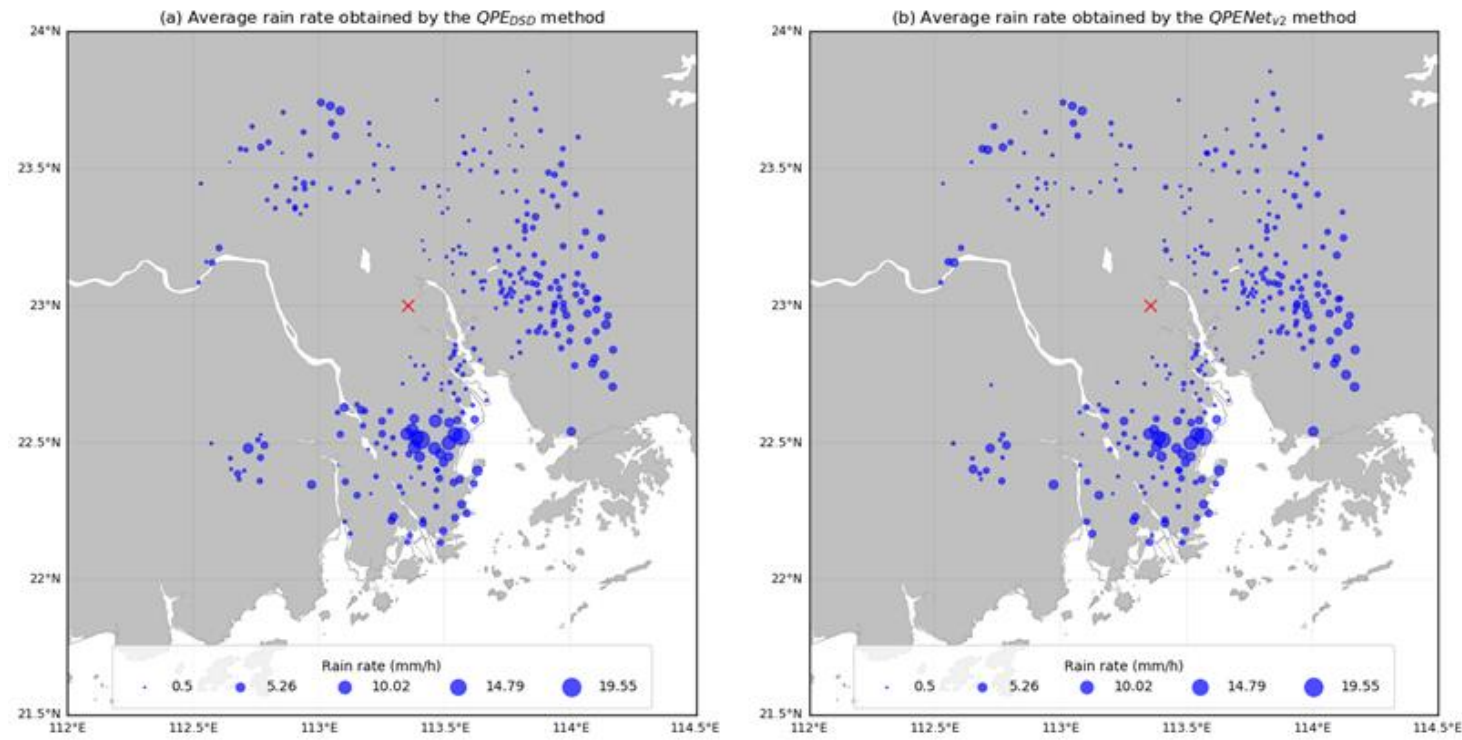

Figure 6. Hourly rainfall estimates using (a) QPE ${ }_{\mathrm{DSD}}$ and (b) QPENet $_{\mathrm{v} 2}$ during typhoon Merbok. The red cross represents the location of the Guangzhou radar.
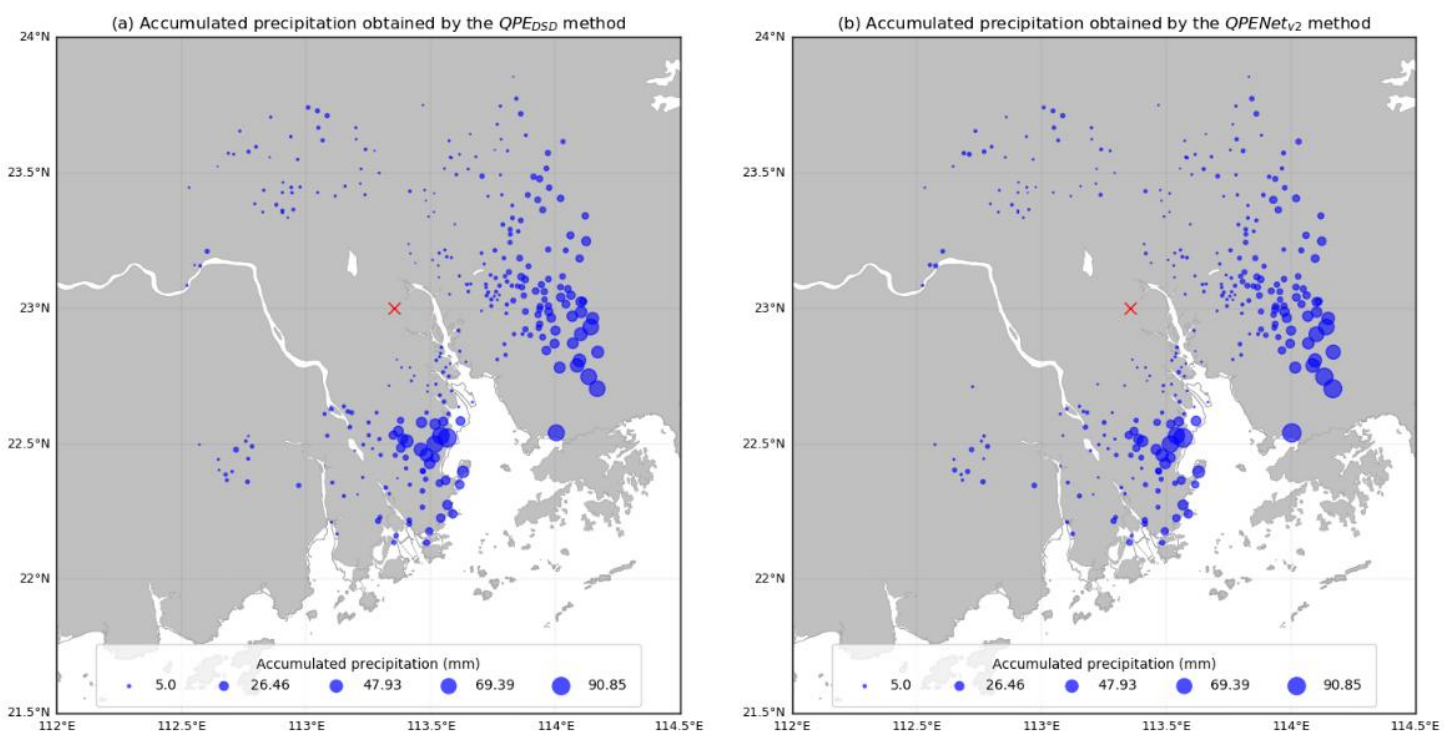

Figure 7. The same as Figure 6, but for the accumulated rainfall during the whole event. 


\subsection{Performance of the QPENet Algorithm}

Table 1 shows the eleven typhoon events that occurred in South China. The evaluated scores on four kinds of QPE algorithms for all rainfall intensities for the Merbok (1702) event are shown in Table 5, the scores for the rainfall intensity less than $5 \mathrm{~mm} \cdot \mathrm{h}^{-1}$ are shown in Table 6, the scores for the rainfall intensity greater than or equal to $5 \mathrm{~mm} \cdot \mathrm{h}^{-1}$ and less than $30 \mathrm{~mm} \cdot \mathrm{h}^{-1}$ are shown in Table 7 , and the scores for the rainfall intensity greater than or equal to $30 \mathrm{~mm}$ are shown in Table 8. The four kinds of QPE algorithms are

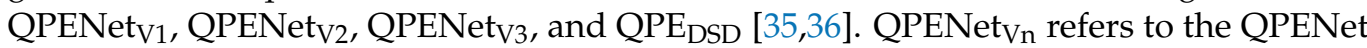
deep-learning model trained with the dataset of version $n(n=1,2$, or 3$)$. QPE $\mathrm{DSD}_{\text {refers to }}$ the QPE algorithm based on DSD fitting.

Table 5. Evaluated scores for four kinds of QPE algorithms for the Merbok event for all rainfall intensities.

\begin{tabular}{cccccc}
\hline QPE Algorithm & CC & RMSE & NB (\%) & NE (\%) & Bias Ratio \\
\hline QPENet $_{\mathrm{V} 1}$ & 0.93 & 2.15 & 4.14 & 38.05 & 1.04 \\
QPENe $_{\mathrm{V} 2}$ & 0.95 & 1.75 & 7.32 & 33.92 & 1.07 \\
QPENet $_{\mathrm{V} 3}$ & 0.96 & 1.97 & -19.42 & 36.72 & 0.81 \\
QPE $_{\mathrm{DSD}}$ & 0.94 & 2.87 & -15.27 & 41.11 & 0.85 \\
\hline
\end{tabular}

Table 6. The same as Table 5 except that the rainfall intensity is less than $5 \mathrm{~mm} \cdot \mathrm{h}^{-1}\left(R<5 \mathrm{~mm} \cdot \mathrm{h}^{-1}\right)$.

\begin{tabular}{cccccc}
\hline QPE Algorithm & CC & RMSE & NB (\%) & NE (\%) & Bias Ratio \\
\hline QPENet $_{\mathrm{V} 1}$ & 0.78 & 0.96 & 22.25 & 45.10 & 1.22 \\
QPENet $_{\mathrm{V} 2}$ & 0.81 & 0.87 & 22.54 & 42.35 & 1.23 \\
QPENet $_{\mathrm{V} 3}$ & 0.74 & 0.84 & -14.14 & 41.58 & 0.86 \\
QPE $_{\mathrm{DSD}}$ & 0.68 & 1.17 & -2.83 & 44.42 & 0.97 \\
\hline
\end{tabular}

Table 7. The same as Table 5 except that the rainfall intensity is greater than or equal to $5 \mathrm{~mm}$ and less than $30 \mathrm{~mm}\left(5 \leq R<30 \mathrm{~mm} \cdot \mathrm{h}^{-1}\right)$.

\begin{tabular}{cccccc}
\hline QPE Algorithm & CC & RMSE & NB (\%) & NE (\%) & Bias Ratio \\
\hline QPENet $_{\mathrm{V} 1}$ & 0.70 & 3.92 & -5.22 & 29.05 & 0.95 \\
QPENe $2_{\text {V }}$ & 0.74 & 3.67 & -1.17 & 25.59 & 0.99 \\
QPENet $_{\mathrm{V} 3}$ & 0.76 & 4.10 & -22.74 & 32.70 & 0.77 \\
QPE $_{\mathrm{DSD}}$ & 0.77 & 5.37 & -26.94 & 36.97 & 0.73 \\
\hline
\end{tabular}

Table 8. The same as Table 5 except that the rainfall intensity is greater than or equal to $30 \mathrm{~mm}$ $\left(R \geq 30 \mathrm{~mm} \cdot \mathrm{h}^{-1}\right)$.

\begin{tabular}{cccccc}
\hline QPE Algorithm & CC & RMSE & NB (\%) & NE (\%) & Bias Ratio \\
\hline QPENet $_{\mathrm{V} 1}$ & 1.00 & 22.03 & -35.88 & 35.88 & 0.64 \\
QPENe $_{\mathrm{V} 2}$ & 0.98 & 16.01 & -24.89 & 24.89 & 0.75 \\
QPENet $_{\mathrm{V} 3}$ & 0.98 & 19.24 & -29.63 & 29.63 & 0.70 \\
QPE $_{\mathrm{DSD}}$ & 0.96 & 30.02 & -37.67 & 37.67 & 0.62 \\
\hline
\end{tabular}

From Tables 5-7, it is found that the QPENet algorithms had better estimation performance than the QPE ${ }_{D S D}$ algorithm, whether for the process with $5 \leq R<30$ and $R \geq 30 \mathrm{~mm} \cdot \mathrm{h}^{-1}$ or for all the rainfall intensities. Only when $R<5 \mathrm{~mm} \cdot \mathrm{h}^{-1}$, the underestimation of the QPE $E_{D S D}$ algorithm was small, and the overestimation of the QPENet algorithm was large. However, the RMSE of QPENet was small and the algorithm was relatively stable. Considering that weak precipitation has a small impact on daily life, these two algorithms are both applicable.

The evaluation indicators of the QPENet and QPE ${ }_{\mathrm{DSD}}$ algorithms were analyzed as follows. For all the rainfall intensities, the CC, RMSE, NB, NE, and bias ratio were increased 
by $0.2 \%, 39 \%, 73 \%, 17 \%$, and $73 \%$ at the most, respectively. When $R$ was greater than or equal to $5 \mathrm{~mm} \cdot \mathrm{h}^{-1}$ and less than $30 \mathrm{~mm} \cdot \mathrm{h}^{-1}$, the CC, RMSE, NB, NE, and bias ratio could be increased by $-1 \%, 32 \%, 96 \%, 31 \%$, and $96 \%$ at the most, respectively. When $R$ was greater than or equal to $30 \mathrm{~mm} \cdot \mathrm{h}^{-1}$, the CC, RMSE, NB, NE, and bias ratio were increased by $4 \%, 47 \%, 34 \%, 34 \%$, and $34 \%$ at the most, respectively. For the three situations of rainfall intensity above, the QPENet algorithms had apparent advantages. However, when $R$ was less than $5 \mathrm{~mm} \cdot \mathrm{h}^{-1}$, the CC, RMSE, NB and bias ratio were increased by $19 \%, 28 \%, 6 \%$, and $-37 \%$ at the most, respectively. The performance of the QPE ${ }_{D S D}$ algorithm was slightly better, indicating that the QPENet algorithm still has room for improvement when there is weak precipitation.

\subsection{Effect of Input Data on the Performance of the QPENet Algorithms}

During the rainfall episode of landfall typhoons, the wind is relatively strong. When establishing the corresponding relationship between dual-polarization quantities and surface automatic weather station observations, the influence of wind needs to be considered. Therefore, according to the distance from the surface automatic weather stations, this study established three versions of the QPE dataset: version 1 was $1.5 \mathrm{~km}$ away from the surface rainfall observation station, version 2 was $3 \mathrm{~km}$ away from the rainfall station, and version 3 was $5 \mathrm{~km}$ away from the rainfall station. After the QPENet model training for the three versions of the dataset, three precipitation estimation models of QPENet ${ }_{V 1}$, QPENet $_{\mathrm{V} 2}$, and QPENet ${ }_{\mathrm{V} 3}$ were obtained. According to Tables 5-8, QPENet $_{\mathrm{V} 2}$ had the best comprehensive estimation performance on all the rainfall intensities, $5 \leq R<30 \mathrm{~mm} \cdot \mathrm{h}^{-1}$ and $R \geq 30 \mathrm{~mm} \cdot \mathrm{h}^{-1}$. It shows that radar observations $3 \mathrm{~km}$ away from the stations were the most relevant to surface precipitation observations in the three cases above. When hourly rainfall intensity was less than $5 \mathrm{~mm}$, QPENet ${ }_{\mathrm{V} 3}$ had the best estimation performance out of the three deep-learning algorithms. This may have been because the position of light rain falling to the surface is uncertain, and lighter rain has smaller drops that are advected further between the height of the radar beam and the surface.

\section{Performance Comparisons between the $\mathrm{QPE}_{\mathrm{DSD}}$ and QPENet ${ }_{\mathrm{V} 2}$ Algorithms}

According to the analysis in Section 4, the QPENet ${ }_{\mathrm{V} 2}$ algorithm had the best comprehensive performance among the three trained deep-learning models. This section compares the performance of $\mathrm{QPENet}$ 22 and the traditional QPE $\mathrm{DSD}_{\mathrm{D}}$ method in detail in three aspects.

\subsection{Performance of $Q P E_{D S D}$ and $Q P E N e t_{V 2}$ under Different Rainfall Intensities}

The scatterplots of the radar rainfall estimates from QPE ${ }_{\mathrm{DSD}}$ and QPENet ${ }_{\mathrm{V} 2}$ versus AWS measurements under different rainfall intensities are shown in Figure 8. When $R<5 \mathrm{~mm} \cdot \mathrm{h}^{-1}$, the estimation accuracy of QPE $\mathrm{DSD}_{\mathrm{D}}$ was slightly better than that of QPENet $\mathrm{V}_{2}$. It may have been due to the relatively large measurement uncertainty of the polarimetric radar variables $\left(Z_{D R}\right.$ and $\left.K_{D P}\right)$ during light rain. Since the QPE $E_{D S D}$ algorithm was based on the segmentation of rain intensity, which takes into account the distribution of light rain, it showed a slightly better performance. However, for all the other rainfall intensities, the QPENet $_{\mathrm{V} 2}$ algorithm had obvious advantages. 
(a)

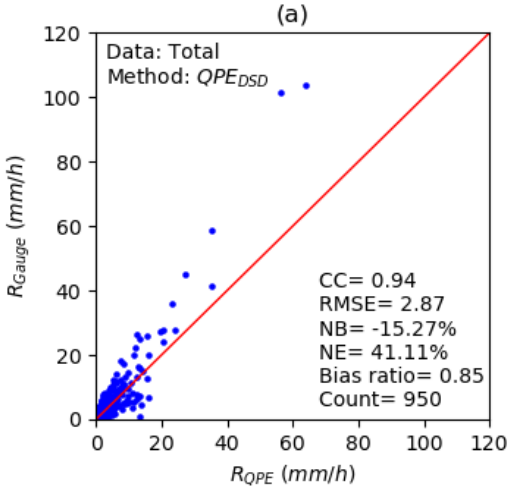

(b)

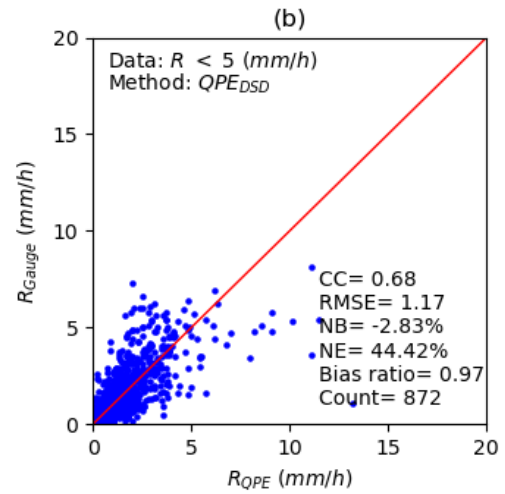

(c)

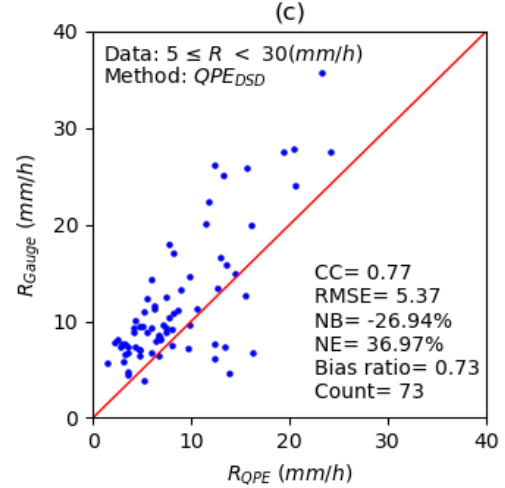

(d)

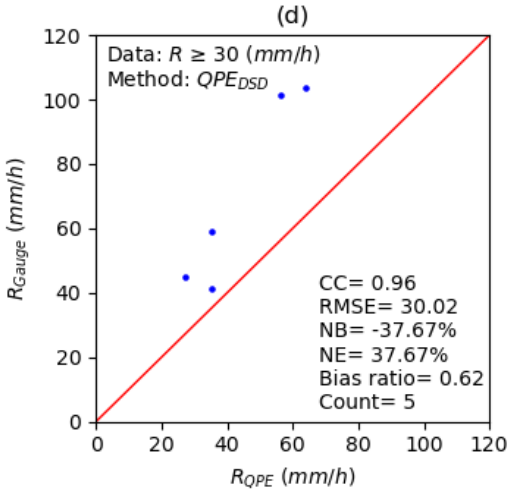

(e)

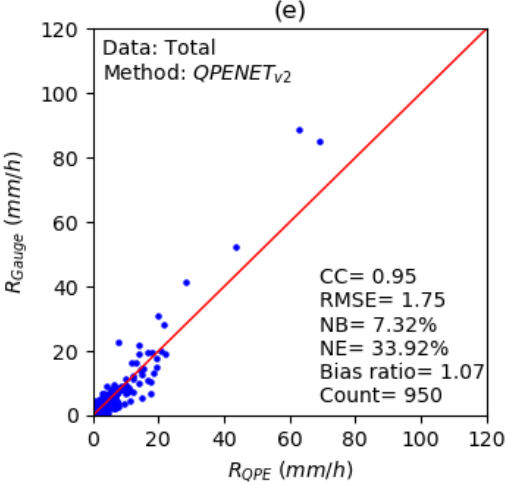

(f)

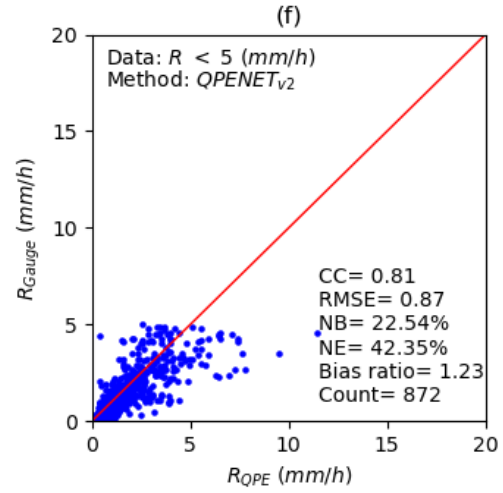

(g)

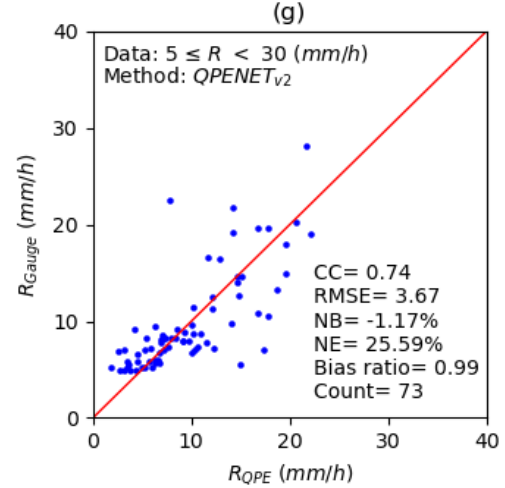

(h)

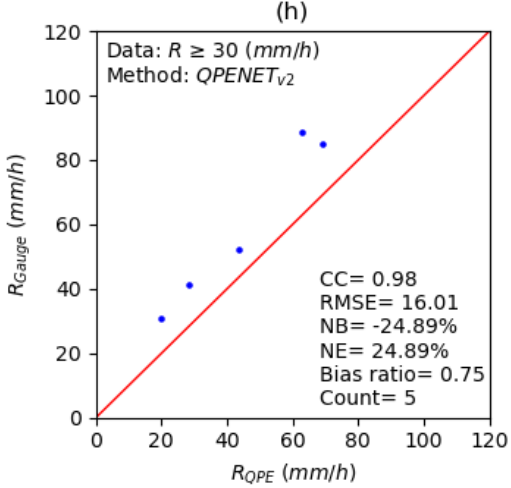

Figure 8. Scatterplots of rainfall estimates from the automatic weather stations versus radar during typhoon Merbok. (a-d) QPE $\mathrm{DSD}_{\text {. }}$. (e-h) QPENet $\mathrm{V}_{\mathrm{V} 2}$. (a,e), (b,f), (c,g), and $(\mathbf{d}, \mathbf{h})$ correspond to all rainfall intensities, $R<5 \mathrm{~mm} \cdot \mathrm{h}^{-1}, 5 \leq R<30 \mathrm{~mm} \cdot \mathrm{h}^{-1}$, and $30 \mathrm{~mm} \cdot \mathrm{h}^{-1} \leq R$, respectively.

5.2. Performance of $Q P E_{D S D}$ and $Q P E N e t_{V 2}$ on Different Segments of $Z_{H}, Z_{D R}$, and $K_{D P}$

The bias ratio and RMSE of the derived rainfall estimates using the QPE ${ }_{D S D}$ and QPENet $_{\mathrm{V} 2}$ algorithms were compared for different segments of $Z_{\mathrm{H}}$ and $Z_{\mathrm{DR}}$ (Figure 9). When $Z_{\mathrm{H}}$ was small $(<20 \mathrm{dBZ})$, the bias ratios of the two algorithms first increased and 
then decreased as $Z_{\mathrm{DR}}$ increased, and the bias ratio of QPENet ${ }_{\mathrm{V} 2}$ decreased faster. When $Z_{\mathrm{H}}$ was moderate (20-30 dBZ), the bias ratios increased with $Z_{\mathrm{DR}}$, and the bias ratio of QPENet $_{\mathrm{V} 2}$ increased slower. When $Z_{\mathrm{H}}$ was large $(\geq 30 \mathrm{dBZ})$, the bias ratio of QPE $\mathrm{DSD}_{\text {first }}$ increased and then decreased with the increase in $Z_{\mathrm{DR}}$, while the bias ratio of QPENet $t_{\mathrm{V} 2}$ decreased with the increase in $Z_{\mathrm{DR}}$.
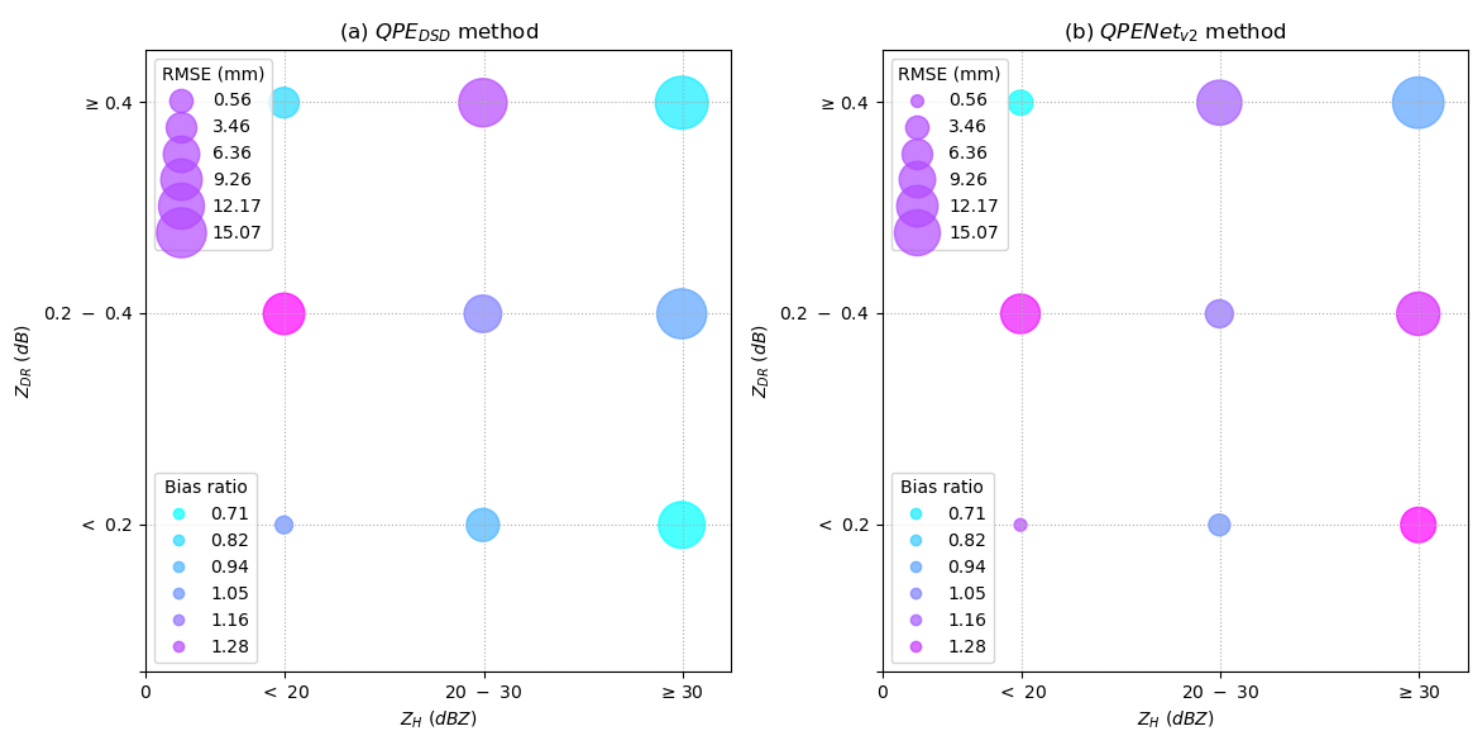

Figure 9. Bubble charts of the bias ratios and RMSEs of the radar-derived hourly rainfall estimates during typhoon Merbok: (a) $\mathrm{QPE}_{\mathrm{DSD}}$; (b) QPENet $\mathrm{V}_{2}$. The bias ratios and RMSEs were calculated for different $Z_{\mathrm{H}}$ and $Z_{\mathrm{DR}}$ ranges using observations from the automatic weather stations as references. $Z_{\mathrm{H}}$ is divided into " $<20 \mathrm{dBZ}$ ", "20-30 dBZ", and " $\geq 30 \mathrm{dBZ}$ ". $Z_{\mathrm{DR}}$ is divided into " $<0.2 \mathrm{~dB}^{\prime}$, , $0.2-0.4 \mathrm{~dB}^{\prime}$, and " $\geq 0.4 \mathrm{~dB}^{\prime}$. The size of the dots represents the RMSE, the color of the dots indicates the bias ratio.

Overall, the RMSE of QPENet ${ }_{\mathrm{V} 2}$ was smaller than that of $\mathrm{QPE}_{\mathrm{DSD}}$, indicating that the former was more stable. But the RMSEs of both algorithms in the upper right corner were rather large, which means when $Z_{\mathrm{H}}$ and $Z_{\mathrm{DR}}$ are large, the stability of both algorithms has room for improvement.

On specific segments of $Z_{\mathrm{H}}$ and $K_{\mathrm{DP}}$, the bias ratio and RMSE of precipitation estimates from QPE $_{\mathrm{DSD}}$ and QPENet ${ }_{\mathrm{V} 2}$ are shown in Figure 10. It can be seen that when $Z_{\mathrm{H}}$ was small

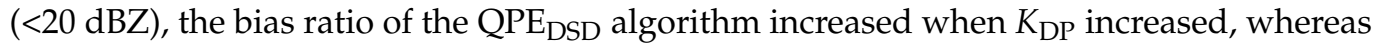
the bias ratio of the QPENet ${ }_{\mathrm{V} 2}$ algorithm first increased and then decreased with the increase in $K_{\mathrm{DP}}$. When $Z_{\mathrm{H}}$ was moderate (20-30 dBZ), the bias ratios of the two algorithms increased with the increase in $K_{\mathrm{DP}}$. When $Z_{\mathrm{H}}$ was large ( $\geq 30 \mathrm{dBZ}$ ), the bias ratios of the two algorithms decreased with the increase in $K_{\mathrm{DP}}$.

However, overall, the RMSE of the QPENet ${ }_{\mathrm{V} 2}$ algorithm was smaller than that of QPE $E_{D S D}$. In particular, when $Z_{\mathrm{H}}$ was small or moderate, the RMSEs of the two algorithms were both small. When $Z_{\mathrm{H}}$ was large, the RMSEs of the two algorithms increased, and the increase in the $\mathrm{QPE}_{\mathrm{DSD}}$ algorithm was more significant. Similar to the $Z_{\mathrm{H}}$ and $Z_{\mathrm{DR}}$ segmentation, the RMSEs of the two algorithms were both large when $Z_{\mathrm{H}}$ and $K_{\mathrm{DP}}$ were large, suggesting that the stability of both algorithms could be improved. 

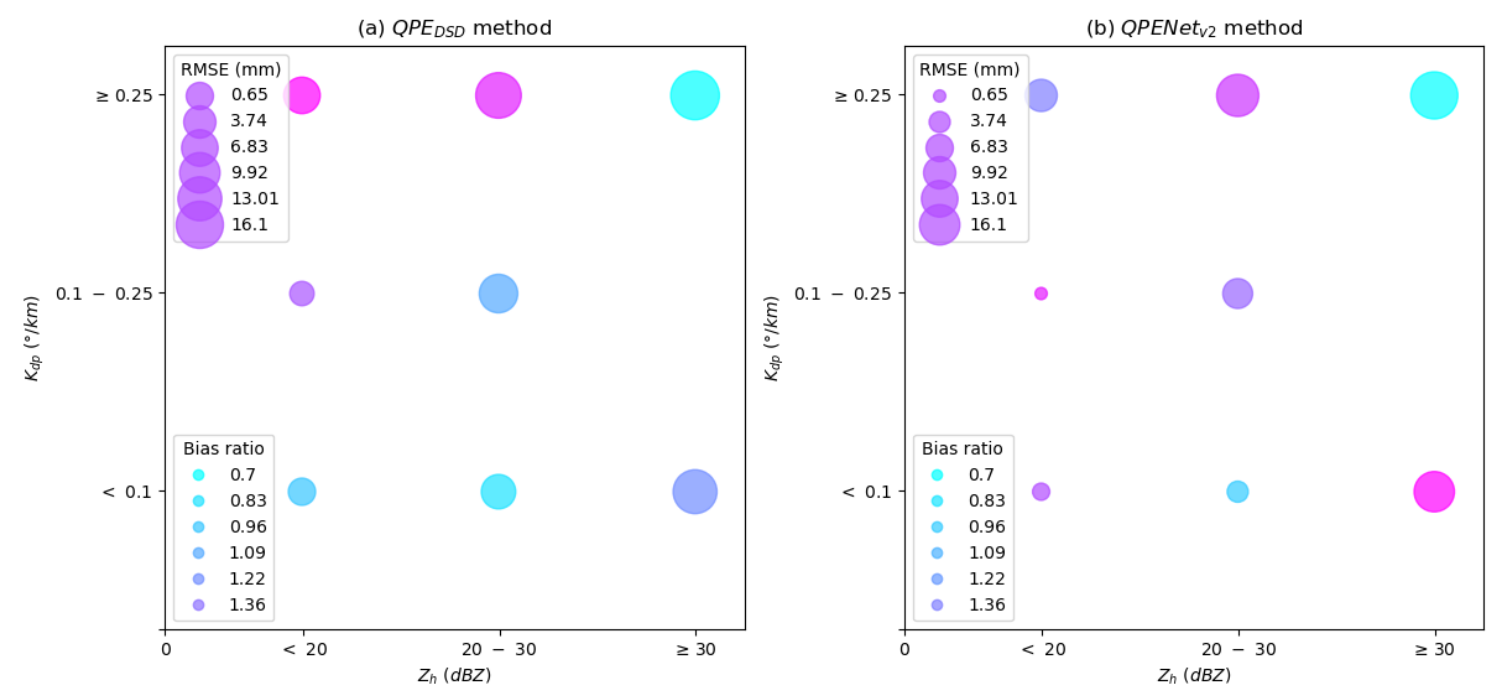

Figure 10. Bubble charts of the bias ratios and RMSEs of the radar-derived hourly rainfall estimates during typhoon Merbok: (a) $\mathrm{QPE}_{\mathrm{DSD}}$; (b) QPENet $\mathrm{V}_{\mathrm{V}}$. The bias ratios and RMSEs were calculated for different $Z_{\mathrm{H}}$ and $K_{\mathrm{DP}}$ ranges using observations from the automatic weather stations as references. $Z_{\mathrm{H}}$ is divided into " $<20 \mathrm{dBZ}$ ", "20-30 dBZ", and " $\geq 30 \mathrm{dBZ}$ ". $K_{\mathrm{DP}}$ is divided into " $<0.1^{\circ} \cdot \mathrm{km}^{-1}$ ", " $0.1-0.25^{\circ} \cdot \mathrm{km}^{-1}$ ", and " $\geq 0.25^{\circ} \cdot \mathrm{km}^{-1}$ ”. The size of the dots represents the RMSE, the color of the dots indicates the bias ratio.

\subsection{Spatial Distribution of the Errors Associated with $Q P E_{D S D}$ and $Q P E N e t_{V 2}$}

The performance of QPE $\mathrm{DSD}$ and QPENet $\mathrm{V}_{\mathrm{V}}$ was further analyzed in terms of the spatial distribution of the bias ratio and RMSE of the estimated precipitation (Figure 11). As can be seen, the large bias ratios were mainly distributed around $22.4^{\circ} \mathrm{N} 112.75^{\circ} \mathrm{E}$, which is due to the partial beam blockage of the Guangzhou radar (i.e., the beam in this area is blocked by an iron tower). The large RMSE areas were mainly distributed in two areas near $22.5^{\circ} \mathrm{N} 113.5^{\circ} \mathrm{E}$ and $23.1^{\circ} \mathrm{N} 114^{\circ} \mathrm{E}$, where the intense rainfall was located (see Figure 6). Overall, the bias ratios and RMSEs of the QPENet ${ }_{\mathrm{V} 2}$ algorithm were smaller than those of $\mathrm{QPE}_{\mathrm{DSD}}$. Especially around $22.6^{\circ} \mathrm{N} 113.25^{\circ} \mathrm{E}$, the bias ratio of QPENet $\mathrm{V}_{2}$ was much smaller. In addition, the RMSE of QPENet $\mathrm{V}_{2}$ was much smaller around $23.1^{\circ} \mathrm{N} 114^{\circ} \mathrm{E}$, further demonstrating that the QPENet ${ }_{\mathrm{V} 2}$ algorithm had better performance.
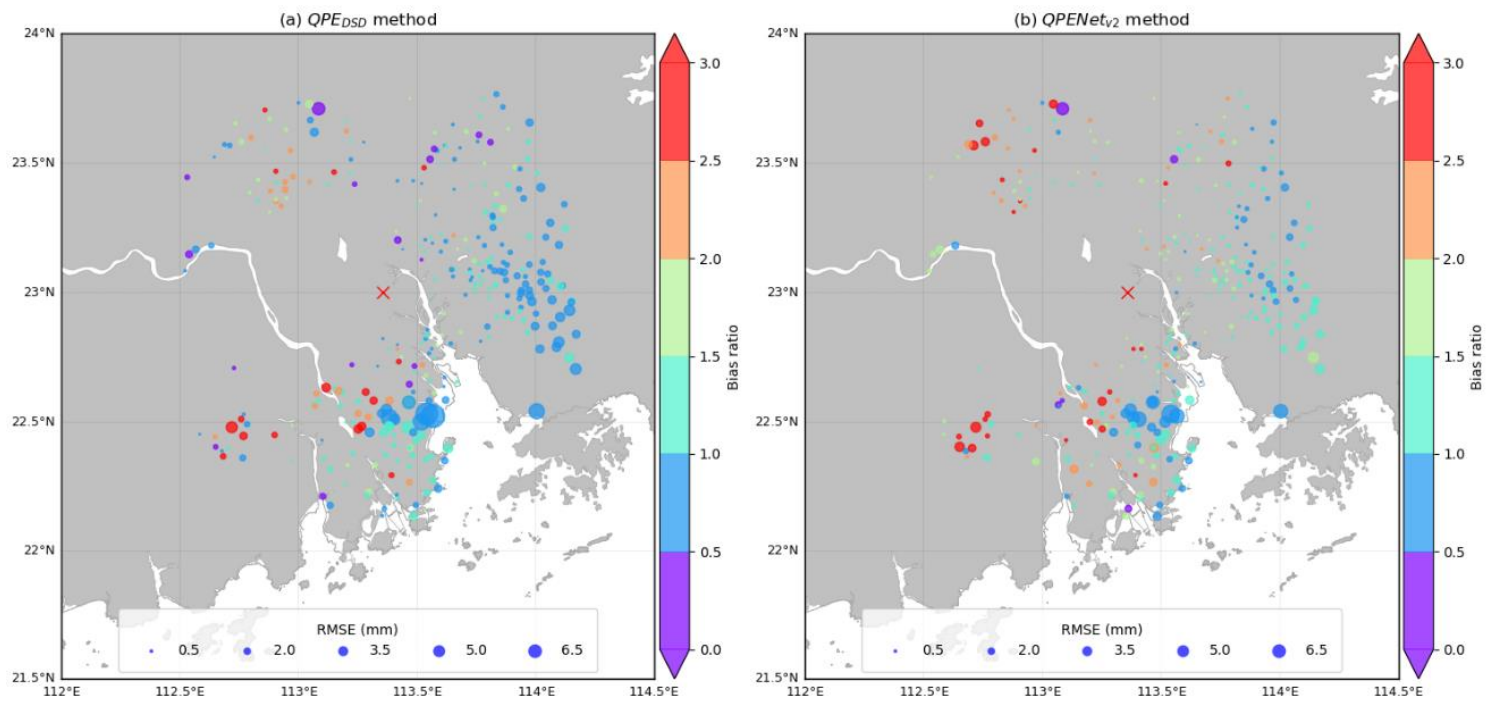

Figure 11. The spatial distributions of the bias ratios and RMSEs of hourly rainfall estimates from (a) QPE $\mathrm{DSD}_{\text {and }}$ (b) QPENet $t_{V 2}$ during typhoon Merbok. The bias ratios and RMSEs were calculated based on the automatic weather station observations. The red cross represents the location of the Guangzhou radar. The size of the dots represents the RMSE, the color of the dots indicates the bias ratio. 


\section{Concluding Remarks}

For this study, we designed a deep neural network algorithm termed QPENet for polarimetric radar QPE. To train this deep-learning model, three versions of the "DPO-SR" dataset were constructed using observations from the radar and AWS during eleven typhoon events in South China. Through evaluation and comparison with the traditional QPE $E_{\mathrm{DSD}}$ method using the test event, the performance and applicability of QPENet were investigated. In order to quantify the influence of the radar observation range on QPE, three datasets of "DPO-SR" were constructed based on the radar observational areas aloft. In particular, $13 \times 13,25 \times 25$, and $41 \times 41$ radar range bins centered by the range bin right on top of the AWS were used to match the AWS measurements in constructing the three datasets. Accordingly, three versions of the QPENet model were then trained. The primary findings are summarized as follows.

(1) Based on the evaluation results for all the rainfall intensities during typhoon Merbok, the new QPENet method has better performance than QPE $_{\mathrm{DSD}}$. The overall CC, RMSE, NB, NE, and bias ratio improved by $0.2 \%, 39 \%, 73 \%, 17 \%$, and $73 \%$, respectively, demonstrating the promising performance of the proposed algorithm.

(2) The evaluation results for different (hourly) rainfall intensities during typhoon Merbok also show that QPENet is superior to QPE ${ }_{D S D}$ for most rain intensities. When $5 \mathrm{~mm} \cdot \mathrm{h}^{-1} \leq R<30 \mathrm{~mm} \cdot \mathrm{h}^{-1}$, the CC, RMSE, NB, NE, and bias ratio improved by $-1 \%, 32 \%$, $96 \%, 31 \%$, and $96 \%$, respectively. When $R \geq 30 \mathrm{~mm} \cdot \mathrm{h}^{-1}$, the CC, RMSE, NB, NE, and bias ratio improved by $4 \%, 47 \%, 34 \%, 34 \%$, and $34 \%$, respectively. Only when $R<5 \mathrm{~mm} \cdot \mathrm{h}^{-1}$, the $\mathrm{QPE}_{\mathrm{DSD}}$ algorithm performed similarly to QPENet, which was likely due to the large measurement uncertainty of $Z_{\mathrm{DR}}$ and $K_{\mathrm{DP}}$ in weak precipitation.

(3) Among the three versions of QPENet, QPENet ${ }_{\mathrm{V} 2}$ has the best overall performance, suggesting that the areal radar observations within $\sim 3 \mathrm{~km}$ from the AWS location can represent the pointwise surface precipitation the best. Although more experiments are required to further demonstrate this, we can interpret such results from two aspects: on the one hand, during the precipitation particle falling processes, the particle location drift from the radar range gate aloft to the surface can be as large as several kilometers, especially when the wind is strong. Using a $3 \mathrm{~km}$ area could resolve the drift fairly well (better than a $1.5 \mathrm{~km}$ area); on the other hand, we should not use an area that is too large to capture the fine structure of precipitation. Based on the results in this study, we conclude that $5 \mathrm{~km}$ may be too large to represent high-intensity precipitation gradients, resulting in reduced performance in rainfall-mapping heavy-rain regions. However, when $R<5 \mathrm{~mm} \cdot \mathrm{h}^{-1}$, the QPENet $_{\mathrm{V} 3}$ algorithm performed the best because light rain falls to the surface in a wider area due to the wind effect. In this case, surface precipitation is more correlated with areal radar observations within $\sim 5 \mathrm{~km}$ from the AWS.

(4) The performance of the QPE $E_{\mathrm{DSD}}$ and QPENet $\mathrm{V}_{\mathrm{V} 2}$ algorithms was analyzed by using the bias ratio and RMSE on different segments of $Z_{\mathrm{H}}, Z_{\mathrm{DR}}$, and $K_{\mathrm{DP}}$. Overall, in most cases, both Figures 9 and 10 show that the bias ratios of QPENet ${ }_{V 2}$ were smaller than those of $\mathrm{QPE}_{\mathrm{DSD}}$. Only when $Z_{\mathrm{H}}$ was large and $Z_{\mathrm{DR}}$ or $K_{\mathrm{DP}}$ was small, the bias ratios of QPE $E_{\mathrm{DSD}}$ were slightly smaller than those of QPENet ${ }_{\mathrm{V} 2}$. Figures 9 and 10 show that the RMSEs of QPENet ${ }_{V 2}$ were smaller than those of QPE ${ }_{\mathrm{DSD}}$ in both Figures 9 and 10; only when $Z_{\mathrm{H}}$, $Z_{D R}$, and $K_{D P}$ were large, the RMSEs of the two algorithms were both large, indicating that there is room for improvement in the stability of the two algorithms. In general, the QPENet $_{\mathrm{V} 2}$ algorithm is better than QPE $\mathrm{DSD}_{\text {. }}$

(5) The performance of the QPE ${ }_{\mathrm{DSD}}$ and $\mathrm{QPENet}_{\mathrm{V} 2}$ algorithms was further investigated by using the spatial distribution of the bias ratios and RMSEs. In the area around $22.4^{\circ} \mathrm{N} 112.75^{\circ} \mathrm{E}$ in Figure 11, the bias ratios of the two methods were both large, which may have been caused by the partial beam blockage of the radar. Near $22.5^{\circ} \mathrm{N} 113.5^{\circ} \mathrm{E}$ and $23.1^{\circ} \mathrm{N} 114^{\circ} \mathrm{E}$, the RMSE was larger, which was caused by the larger hourly rainfall intensity (Figure 6). In general, the bias ratio and RMSE of the QPENet ${ }_{V 2}$ algorithm were both smaller and its performance was better than those of QPE ${ }_{\mathrm{DSD}}$. 
In summary, the QPENet algorithm has a better performance to estimate surface rainfall intensity from polarimetric radar observations. When precipitation intensity is greater than or equal to $30 \mathrm{~mm} / \mathrm{h}$, the advantage of the QPENet algorithm is especially obvious. Nevertheless, the current QPENet algorithm is greatly affected by the quality of radar data. For example, when the rain intensity is less than $5 \mathrm{~mm} \cdot \mathrm{h}^{-1}$, the observational error of $Z_{\mathrm{DR}}$ and $K_{\mathrm{DP}}$ may be large, resulting in a large bias ratio of the QPENet algorithm. In addition, the limited amount of training and test data could have a negative impact on the performance of the QPENet algorithm. To address this, we need to extend the application to cover a variety of severe precipitation events for both training and testing.

Author Contributions: Conceptualization, L.L., S.B. and Y.Z. (Yonghua Zhang); methodology, L.L., Y.Z. (Yonghua Zhang) and H.C.; formal analysis, Y.Z. (Yonghua Zhang), P.S. and Y.Z. (Yang Zhang); resources, Y.Z. (Yi Zhang); data curation, F.Y.; writing—original draft preparation, Y.Z. (Yonghua Zhang), H.C. and P.S.; writing-review and editing, H.C., S.B. and S.Y.; project administration, Y.W.; visualization, Y.Z. (Yonghua Zhang); validation, Y.Z. (Yang Zhang), funding acquisition, Y.Z. (Yi Zhang). All authors have read and agreed to the published version of the manuscript.

Funding: This research was jointly funded by the Key-Area Research and Development Program of Guangdong Province (grant No. 2020B0101130021), the National Key R\&D Program of China (grant Nos. 2018YFC1507401 and 2019YFC1510203), and the National Natural Science Foundation of China (grant No. 41971340). The work of H.C. and S.Y. was supported by Colorado State University.

Institutional Review Board Statement: Not applicable.

Informed Consent Statement: Not applicable.

Data Availability Statement: Not applicable.

Acknowledgments: We would like to thank Guangdong Meteorological Service for collecting and archiving the dual-polarization radar and automatic weather station data used in this study.

Conflicts of Interest: The authors declare no conflict of interest.

\section{References}

1. Yin, J.; Yin, Z.; Xu, S. Composite risk assessment of typhoon-induced disaster for China's coastal area. Nat. Hazards 2013, 69, 1423-1434. [CrossRef]

2. Bringi, V.N.; Chandrasekar, V. Polarimetric Doppler Weather Radar: Principles and Applications; Cambridge University Press: Cambridge, UK, 2001; pp. 635-636.

3. Chen, H.; Chandrasekar, V.; Tan, H.; Cifelli, R. Rainfall Estimation From Ground Radar and TRMM Precipitation Radar Using Hybrid Deep Neural Networks. Geophys. Res. Lett. 2019, 46, 10669-10678. [CrossRef]

4. Cifelli, R.; Chandrasekar, V.; Chen, H.; Johnson, L.E. High Resolution Radar Quantitative Precipitation Estimation in the San Francisco Bay Area: Rainfall Monitoring for the Urban Environment. J. Meteorol. Soc. Jpn. 2018, 96, 141-155. [CrossRef]

5. Gou, Y.; Ma, Y.; Chen, H.; Wen, Y. Radar-derived quantitative precipitation estimation in complex terrain over the eastern Tibetan Plateau. Atmos. Res. 2018, 203, 286-297. [CrossRef]

6. Xia, Q.; Zhang, W.; Chen, H.; Lee, W.-C.; Han, L.; Ma, Y.; Liu, X. Quantification of Precipitation Using Polarimetric Radar Measurements during Several Typhoon Events in Southern China. Remote Sens. 2020, 12, 2058. [CrossRef]

7. Ryzhkov, A.V.; Schuur, T.J.; Burgess, D.W.; Heinselman, P.L.; Giangrande, S.E.; Zrnic, D.S. The joint polarization experiment: Polarimetric rainfall measurements and hydrometeor classification. Bull. Am. Meteorol. Soc. 2005, 86, 809-824. [CrossRef]

8. Xiao, R.; Chandrasekar, V. Development of neural network based algorithm for rainfall estimation based on radar measurements. IEEE Trans. Geosci. Remote Sens. 1997, 35, 160-171. [CrossRef]

9. Chen, H.; Chandrasekar, V.; Cifelli, R. A Deep Learning Approach to Dual-Polarization Radar Rainfall Estimation. In Proceedings of the 2019 URSI Asia-Pacific Radio Science Conference (AP-RASC), New Delhi, India, 9-15 March 2019; pp. 1-2. [CrossRef]

10. Xu, G.; Chandrasekar, V. Operational Feasibility of Neural-Network-Based Radar Rainfall Estimation. IEEE Geosci. Remote Sens. Lett. 2005, 2, 13-17. [CrossRef]

11. Vulpiani, G.; Giangrande, S.; Marzano, F.S. Rainfall estimation from polarimetric S-band radar measurements: Validation of a neutral netwrok approach. J. Appl. Meteorol. Climatol. 2009, 48, 2022-2036. [CrossRef]

12. Dolan, B.; Fuchs, B.; Rutledge, S.A.; Barnes, E.; Thompson, E.J. Primary Modes of Global Drop Size Distributions. J. Atmos. Sci. 2018, 75, 1453-1476. [CrossRef]

13. Wen, G.; Chen, H.; Zhang, G.; Sun, J. An Inverse Model for Raindrop Size Distribution Retrieval with Polarimetric Variables. Remote Sens. 2018, 10, 1179. [CrossRef] 
14. Kitchen, M.; Brown, R.; Davies, A.G. Real-time correction of weather radar data for the effects of bright band, range and orographic growth in widespread precipitation. Q. J. R. Meteorol. Soc. 1994, 120, 1231-1254. [CrossRef]

15. Steiner, M.; Smith, J.A. Reflectivity, Rain Rate, and Kinetic Energy Flux Relationships Based on Raindrop Spectra. J. Appl. Meteorol. 2000, 39, 1923-1940. [CrossRef]

16. Kirstetter, P.-E.; Gourley, J.J.; Hong, Y.; Zhang, J.; Moazamigoodarzi, S.; Langston, C.; Arthur, A. Probabilistic precipitation rate estimates with ground-based radar networks. Water Resour. Res. 2015, 51, 1422-1442. [CrossRef]

17. Chen, H.; Chandrasekar, V.; Bechini, R. An Improved Dual-Polarization Radar Rainfall Algorithm (DROPS2.0): Application in NASA IFloodS Field Campaign. J. Hydrometeorol. 2017, 18, 917-937. [CrossRef]

18. Hinton, G.E.; Salakhutdinov, R.R. Reducing the Dimensionality of Data with Neural Networks. Science 2006, 313, 504-507. [CrossRef] [PubMed]

19. LeCun, Y.; Bengio, Y.; Hinton, G. Deep learning. Nature 2015, 521, 436-444. [CrossRef] [PubMed]

20. Han, L.; Zhao, Y.; Chen, H.; Chandrasekar, V. Advancing Radar Nowcasting Through Deep Transfer Learning. IEEE Trans. Geosci. Remote Sens. 2021, in press. [CrossRef]

21. Chen, H.; Chandrasekar, V.; Cifelli, R.; Xie, P. A Machine Learning System for Precipitation Estimation Using Satellite and Ground Radar Network Observations. IEEE Trans. Geosci. Remote Sens. 2019, 58, 982-994. [CrossRef]

22. Tan, H.; Chandra, C.V.; Chen, H. A Deep Neural Network Model for Rainfall Estimation Using Polarimetric WSR-88DP Radar Observations. American Geophysical Union, Fall Meeting, 2016, abstract \#IN11B-1622. Available online: https: / / agu.confex. com/agu/fm16/meetingapp.cgi/Paper/196830 (accessed on 1 June 2021).

23. Tan, H.; Chandrasekar, V.; Chen, H. A Machine Learning Model for Radar Rainfall Estimation Based on Gauge Observations. In Proceedings of the 2017 United States National Committee of URSI National Radio Science Meeting (USNC-URSI NRSM), Montreal, QC, Canada, 19-26 August 2017; pp. 1-2. [CrossRef]

24. Chen, H.; Chandrasekar, V.; Cifelli, R.; Xie, P.; Tan, H. A data fusion system for accurate precipitation estimation using satellite and ground radar observations: Urban scale application in Dallas-Fort Worth Metroplex. In Proceedings of the 2017 XXXIInd General Assembly and Scientific Symposium of the International Union of Radio Science (URSI GASS), Montreal, QC, Canada, 19-26 August 2017; pp. 1-2. [CrossRef]

25. Chandrasekar, V.; Tan, H.; Chen, H. A machine learning system for rainfall estimation from spaceborne and ground radars. In Proceedings of the 2017 XXXIInd General Assembly and Scientific Symposium of the International Union of Radio Science (URSI GASS), Montreal, QC, Canada, 19-26 August 2017; pp. 1-2. [CrossRef]

26. Moraux, A.; Dewitte, S.; Cornelis, B.; Munteanu, A. Deep Learning for Precipitation Estimation from Satellite and Rain Gauges Measurements. Remote Sens. 2019, 11, 2463. [CrossRef]

27. Wu, C.; Liu, L.; Wei, M.; Xi, B.; Yu, M. Statistics-based optimization of the polarimetric radar hydrometeor classification algorithm and its application for a squall line in South China. Adv. Atmos. Sci. 2018, 35, 296-316. [CrossRef]

28. Wang, Y.; Chandrasekar, V. Algorithm for Estimation of the Specific Differential Phase. J. Atmos. Ocean. Technol. 2009, 26, 2565-2578. [CrossRef]

29. Gou, Y.; Liu, L.; Yang, J.; Wu, C. Operational application and evaluation of the quantitative precipitation estimates algorithm based on the multi-radar mosaic. Acta Meteorol. Sin. 2014, 72, 731-748. (In Chinese)

30. Krizhevsky, A.; Sutskever, I.; Hinton, G.E. Imagenet classification with deep convolutional neural networks. Adv. Neural Inf. Process. Syst. 2012, 25, 1097-1105. [CrossRef]

31. Szegedy, C.; Liu, W.; Jia, Y.; Sermanet, P.; Reed, S.; Anguelov, D.; Erhan, D.; Vanhoucke, V.; Rabinovich, A. Going deep-er with convolutions. In Proceedings of the IEEE Conference on Computer Vision and Pattern Recognition, Boston, MA, USA, 7-12 June 2015; pp. 1-9. [CrossRef]

32. He, K.; Zhang, X.; Ren, S.; Sun, J. Deep Residual Learning for Image Recognition. In Proceedings of the IEEE Conference on Computer Vision and Pattern Recognition (CVPR), Las Vegas, NV, USA, 27-30 June 2016; pp. 770-778. [CrossRef]

33. Liu, Y.; Minh Nguyen, D.; Deligiannis, N.; Ding, W.; Munteanu, A. Hourglass-shapenetwork based semantic segmentation for high resolution aerial imagery. Remote Sens. 2017, 9, 522. [CrossRef]

34. Glorot, X.; Bengio, Y. Understanding the difficulty of training deep feedforward neural networks. In Proceedings of the Thirteenth International Conference on Artificial Intelligence and Statistics Intelligence and Statistics, Sardinia, Italy, 13-15 May 2010; pp. 249-256.

35. Zhang, Y.; Liu, L.; Bi, S.; Wu, Z.; Shen, P.; Ao, Z.; Chen, C.; Zhang, Y. Analysis of Dual-Polarimetric Radar Variables and Quantitative Precipitation Estimators for Landfall Typhoons and Squall Lines Based on Disdrometer Data in Southern China. Atmosphere 2019, 10, 30. [CrossRef]

36. Zhang, Y.; Liu, L.; Wen, H.; Wu, C.; Zhang, Y. Evaluation of the Polarimetric-Radar Quantitative Precipitation Estimates of an Extremely Heavy Rainfall Event and Nine Common Rainfall Events in Guangzhou. Atmosphere 2018, 9, 330. [CrossRef] 